\title{
Effects of dose modifications on the safety and efficacy of dacomitinib for EGFR mutation-positive non-small-cell lung cancer
}

\author{
Jesús Corral*,1, Tony S Mok², Kazuhiko Nakagawa ${ }^{3}$, Rafael Rosell ${ }^{4}$, Ki Hyeong Lee ${ }^{5}$, Maria \\ Rita Migliorino ${ }^{6}$, Adam Pluzanski ${ }^{7}$, Rolf Linke ${ }^{8}$, Geeta Devgan ${ }^{9}$, Weiwei $\operatorname{Tan}^{10}$, Susan \\ Quinn ${ }^{11}$, Tao Wang ${ }^{12}$ \& Yi-Long Wu ${ }^{13}$ \\ ${ }^{1}$ Clínica Universidad de Navarra, Madrid, 28027, Spain \\ ${ }^{2}$ State Key Laboratory of South China, Department of Clinical Oncology, Chinese University of Hong Kong, Hong Kong, PR China \\ ${ }^{3}$ Kindai University Hospital, Osaka, 589-8511, Japan \\ ${ }^{4}$ Catalan Institute of Oncology, Barcelona, Spain \\ ${ }^{5}$ Chungbuk National University Hospital, Cheongju, Korea \\ ${ }^{6}$ Pulmonary Oncology Unit, San Camillo-Forlanini Hospital, Rome, 00152, Italy \\ ${ }^{7}$ The Maria Sklodowska-Curie Memorial Cancer Centre \& Institute of Oncology, Warsaw, 02-781, Poland \\ ${ }^{8}$ SFJ Pharmaceuticals ${ }^{\circledR}$, Pleasanton, CA 94588, USA \\ ${ }^{9}$ Pfizer Oncology, New York, NY 10017, USA \\ ${ }^{10}$ Pfizer Clinical Pharmacology, San Diego, CA 92121, USA \\ ${ }^{11}$ Pfizer Oncology, Cambridge, MA 02139, USA \\ ${ }^{12}$ Pfizer Oncology, Groton, CT 06340, USA \\ ${ }^{13}$ Guangdong Lung Cancer Institute, Guangdong Provincial People's Hospital \& Guangdong Academy of Medical Sciences, \\ Guangzhou, Guangdong, 510080, PR China \\ *Author for correspondence: jcorral@unav.es
}

Aim: We evaluated reasons for dacomitinib dose reduction (DR) and examined adverse event (AE) incidence, key efficacy end points (progression-free survival [PFS]/overall survival [OS]), and pharmacokinetics in dose-reducing patients in the ARCHER 1050 trial. Patients \& methods: Newly diagnosed patients with EGFR mutation-positive, advanced non-small-cell lung cancer received oral dacomitinib (45 mg once-daily [QD]), with stepwise toxicity-managing DR (30 and $15 \mathrm{mg}$ QD) permitted. Results: Skin toxicities (62.7\%) were the most common DR-leading AEs. The AE incidence and severity decreased following DRs. Initial plasma dacomitinib exposure (45 mg QD) was generally lower in patients remaining at $45 \mathrm{mg}$ QD compared with dose-reducing patients. Median PFS and OS were similar in all dacomitinib-treated patients and dose-reducing patients. Conclusion: Tolerability-guided dose modifications enabled patients to continue with dacomitinib and benefit from PFS/OS improvement.

Trial registration number: NCT01774721

First draft submitted: 23 May 2019; Accepted for publication: 24 June 2019; Published online: 17 July 2019

Keywords: dacomitinib $\bullet$ dose $\bullet$ EGFR $\bullet$ first-line $\bullet$ NSCLC

EGFR-targeted therapy is a standard treatment for EGFR mutation-positive advanced non-small-cell lung cancer (NSCLC). Dacomitinib is a second-generation, irreversible EGFR tyrosine kinase inhibitor (TKI) that is US FDA-approved for first-line treatment of patients with metastatic NSCLC with EGFR exon 19 deletion or exon 21 L858R substitution mutations [1]. Similar to other second-generation EGFR TKIs, dacomitinib is associated with more potent EGFR inhibition than first-generation EGFR TKIs. This relatively potent EGFR inhibition is associated with improved clinical efficacy. In the ARCHER 1050 trial (NCT01774721), significant improvement was observed with dacomitinib versus the first-generation EGFR TKI gefitinib in both progression-free survival (PFS; hazard ratio [HR]: 0.59; [95\% CI: 0.47-0.74]; $\mathrm{p}<0.0001$; median PFS; 14.7 vs 9.2 months) and overall survival (OS; HR: 0.76; [95\% CI: 0.58-0.99]; $\mathrm{p}=0.044$; median OS; 34.1 vs 26.8 months) [2,3]. However, the relatively potent EGFR inhibition seen with second-generation EGFR TKIs is also associated with EGFRmediated toxicities. In the ARCHER 1050 trial, EGFR-mediated toxicities reported included diarrhea, paronychia, 
dermatitis acneiform and stomatitis. Furthermore, in the ARCHER 1050 trial, 66\% of patients in the dacomitinib arm experienced dose reduction (DR). However, grade 4 adverse events (AEs) occurred in only $2 \%$ of dacomitinibtreated patients in that trial, and only $10 \%$ of dacomitinib-treated patients permanently discontinued the drug due to study drug-related AEs [2]. The overall safety profile of dacomitinib in the ARCHER 1050 trial was similar to that seen with other EGFR TKIs [2]. Tolerability-guided DR has proven to be an effective strategy in reducing treatment-related AEs associated with afatinib, a second-generation EGFR TKI, in a randomized Phase II study without negatively impacting the drug's therapeutic efficacy $[4,5]$.

In the ARCHER 1050 trial, the starting dose of dacomitinib for all patients was $45 \mathrm{mg}$ once daily (QD), with a maximum of two dose-level reductions permitted for treatment-related toxicities. The first DR was to $30 \mathrm{mg}$ QD, while the second was to $15 \mathrm{mg}$ QD. This analysis explored the effects of dacomitinib DR on safety, efficacy and pharmacokinetics (PK) outcomes in the ARCHER 1050 trial.

\section{Patients \& methods}

Trial design \& patients

The ARCHER 1050 trial design and patient eligibility criteria have been published previously $[2,3]$. ARCHER 1050 was an open-label, multinational, multicenter and randomized Phase III trial. Eligible patients had stage IIIB/IV or recurrent, EGFR mutation-positive NSCLC, measurable disease by Response Evaluation Criteria in Solid Tumors version 1.1, and an Eastern Cooperative Oncology Group performance status of 0 or 1 . The primary end point of the trial was PFS as determined by blinded independent central radiologic review. Secondary end points included OS, best overall response, duration of response, overall safety profile, patient-reported outcomes and PK. The data cut-off date was 17 February 2017 for OS and 29 July 2016 for all other end points. The trial was conducted in accordance with legal and regulatory requirements, as well as the general principles set forth in the International Ethical Guidelines for Biomedical Research Involving Human Subjects (Council for International Organizations of Medical Sciences 2002) and the Declaration of Helsinki (World Medical Association 1996 and 2008). All patients provided written informed consent before enrollment.

\section{Treatment}

Patients were randomized (1:1) to oral dacomitinib $45 \mathrm{mg}$ QD in 28-day cycles or oral gefitinib $250 \mathrm{mg}$ QD in 28-day cycles (dosing and schedule details for both arms have been reported previously) [2,3]. Randomization was stratified by EGFR mutation type (exon 19 deletion vs exon 21 L858R substitution) and race (Japanese vs mainland Chinese vs other East Asian vs non-East Asian). All patients randomized to the dacomitinib arm started with the standard $45 \mathrm{mg}$ QD dose as per protocol. Dacomitinib DRs were permitted in order to manage treatment-related toxicity that was not controlled with optimal supportive care, or not tolerated due to symptoms or interference with normal daily activities regardless of severity. If, in the judgment of the investigator, a patient subsequently tolerated treatment well at a reduced dose level, the dose was permitted to be increased to the prior dose level. When implemented, the first dacomitinib DR was always from 45 to $30 \mathrm{mg} \mathrm{QD}$, and the second was always from 30 to $15 \mathrm{mg}$ QD. If a patient could not tolerate treatment after DR to $15 \mathrm{mg}$, treatment was discontinued. For grade 3, grade 4 or intolerable grade 2 toxicity (using Common Terminology Criteria for Adverse Events [CTCAE] v4.0), treatment was interrupted. Upon recovery to grade 2 or baseline, and in the clinical judgment of the investigator with the agreement of the patient, dacomitinib treatment was resumed as follows: for interruption due to grade 3 or intolerable grade 2 toxicity, treatment was permitted to be resumed at the same or a reduced dose level; for episodes of grade 4 toxicity, reduction to the next dose level was mandated. Per the protocol, interruptions to manage AEs were not anticipated to extend beyond 2 weeks. However, as permitted by the protocol, following discussion of the clinical circumstance between the investigator and sponsor, there were individual cases where patients were permitted to resume treatment after a lapse of greater than 2 weeks.

\section{Assessments}

Patients were observed for survival status and subsequent cancer therapies for up to 48 months from the date of the first dose. All causality AEs were measured and graded for severity according to National Cancer Institute CTCAE (NCI-CTCAE) v4.0. Incidence reported included all patients with an AE starting or ongoing during that interval. Skin toxicities was a compound term consisting of two clustered terms: other skin toxicity (dry skin, nail disorder, palmar-plantar erythrodysesthesia syndrome, paronychia, skin fissures, skin ulcer or xerosis) and rash/dermatitis acneiform (any reported preferred term within high-level term acnes plus Medical Dictionary for Regulatory 
Activities [MedDRA] preferred terms, including drug eruption, rash, rash erythematous, rash generalized, rash maculo-papular or rash pruritic). Diarrhea was a clustered term encompassing acute prerenal failure, azotemia, dehydration, diarrhea, blood urea nitrogen/creatinine ratio increased, electrolyte imbalance, hypovolemia and prerenal failure. All other reported AEs were recorded by preferred terms.

\section{Post hoc analyses}

For patients with dacomitinib DRs, frequency and severity of AEs of interest (diarrhea, dermatitis acneiform, stomatitis and paronychia) before and after DR from $45 \mathrm{mg}$ QD were analyzed by NCI-CTCAE grade. Plasma steady-state trough concentrations $\left(\mathrm{C}_{\text {trough, ss }}\right.$ ) of dacomitinib were collected at day 1 of cycle 2 , after at least 14 days of consecutive dacomitinib $45 \mathrm{mg}$ QD dosing. These measurements were used to compare the initial plasma exposure in patients who remained at $45 \mathrm{mg}$ QD for the duration of treatment, patients whose dose was reduced to $30 \mathrm{mg}$ QD as the lowest dose and patients whose dose was reduced to $15 \mathrm{mg}$ QD as the lowest dose. Kaplan-Meier estimates were used to construct survival curves and calculate median (with 95\% CIs) PFS and OS for all patients in the dacomitinib arm, all patients in the dacomitinib arm who underwent DR (to either $30 \mathrm{mg}$ QD or $15 \mathrm{mg}$ QD as lowest dose), patients in the dacomitinib arm who remained at $45 \mathrm{mg}$ QD for the duration of treatment, patients in the dacomitinib arm who dose reduced to $30 \mathrm{mg}$ QD as lowest dose and patients who dose reduced to $15 \mathrm{mg}$ QD as lowest dose.

\section{Results}

\section{Patients}

Among all patients starting on the 45-mg QD dose of dacomitinib $(\mathrm{n}=227)$, DRs due to AEs occurred in 150 (66.1\%) patients, while 77 (33.9\%) patients stayed at $45 \mathrm{mg}$ QD for the duration of treatment. In total 87 patients (38.3\% of all patients randomized to the dacomitinib arm) reduced to $30 \mathrm{mg}$ QD as the lowest dose and 63 patients $(27.8 \%$ of all patients randomized to the dacomitinib arm) further dose reduced to $15 \mathrm{mg}$ QD as the lowest dose. Median time to DR was 12.9 weeks for the 87 patients with $30 \mathrm{mg}$ QD as the lowest dose received. For the 63 patients who received $15 \mathrm{mg}$ as the lowest dose, median time to the first DR (to $30 \mathrm{mg}$ QD) was 8.4 weeks and median time to subsequent DR to $15 \mathrm{mg}$ QD (from $30 \mathrm{mg}$ QD) was 12.4 weeks. There were no baseline clinical characteristics that were predictive of which patients received DRs. However, DRs generally occurred more frequently in females. Additionally, median weights, as well as the lower- and upper-range bounds for weight, were lower in patients in both DR groups than in those remaining at $45 \mathrm{mg}$ QD for the duration of treatment (Supplementary Table 1).

\section{Treatment exposure}

Among patients treated at $45 \mathrm{mg}$ QD who did not experience DRs $(\mathrm{n}=77)$, the median (range) total treatment time was $36.1(0.3-115.1)$ weeks, as compared with 63.7 (5.1-162.7) weeks for patients with $30 \mathrm{mg}$ QD as the lowest dose ( $n=87)$ and $83.7(11.9-161.7)$ for patients with $15 \mathrm{mg}$ QD as the lowest dose $(n=63)$. These numbers reflect the entire duration of dacomitinib treatment in patients who dose reduced, by lowest dose received, not duration of treatment only at a specific dose level.

\section{Efficacy}

Median PFS was similar in all dacomitinib-treated patients $(\mathrm{n}=227)$ and those with DR $(\mathrm{n}=150): 14.7$ (95\% CI: 11.1-16.6) months and 16.6 (95\% CI: 14.6-18.6) months, respectively (Figure 1A). Median (95\% CI) PFS by the lowest dose received was as follows: $9.1(5.6-12.8)$ months for patients $(\mathrm{n}=77)$ who remained on $45 \mathrm{mg}$ QD and $12.9(10.8-16.7)$ months for patients $(\mathrm{n}=87)$ who received $30 \mathrm{mg}$ QD as the lowest dose and $31.2(16.5-35.1)$ months for patients $(\mathrm{n}=63)$ who received $15 \mathrm{mg}$ QD as the lowest dose (Figure 1B). Median (95\% CI) OS was also similar in all dacomitinib-treated patients $(\mathrm{n}=227)$ and those with DR $(\mathrm{n}=151): 34.1(29.5-37.7)$ months and 36.7 (32.6 - not reached [NR]) months, respectively (Figure 2A). Median (95\% CI) OS for each of the lowest dose groups was as follows: $22.0(15.6-26.4)$ months for patients $(\mathrm{n}=76)$ who remained on $45 \mathrm{mg}$ QD and 32.6 (28.8-37.7) months for patients $(\mathrm{n}=88)$ who received $30 \mathrm{mg}$ QD as the lowest dose and NR (34.7-NR) months for patients $(n=63)$ who received $15 \mathrm{mg}$ QD as the lowest dose (Figure 2B). 
(A)

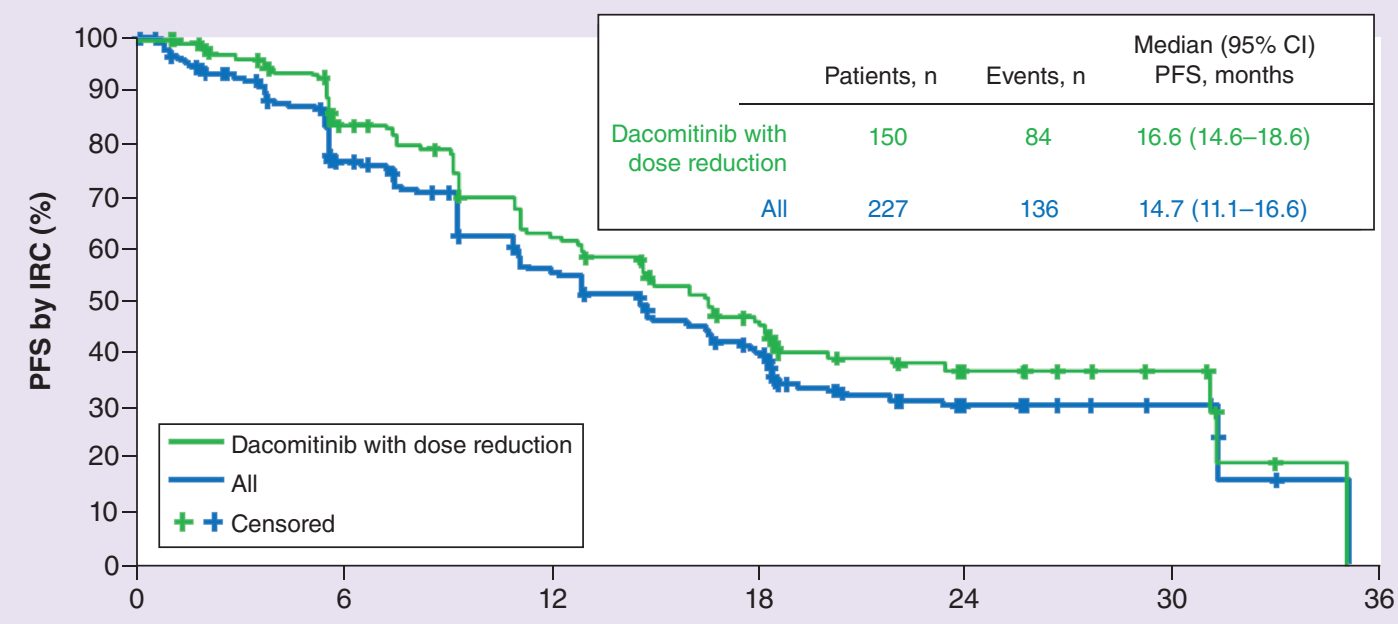

No. at risk:

Dacomitinib with

dose reduction

All 227

150

116

83

Time (months)

(B)

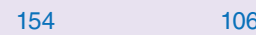

106

57

73
18

20
6

6
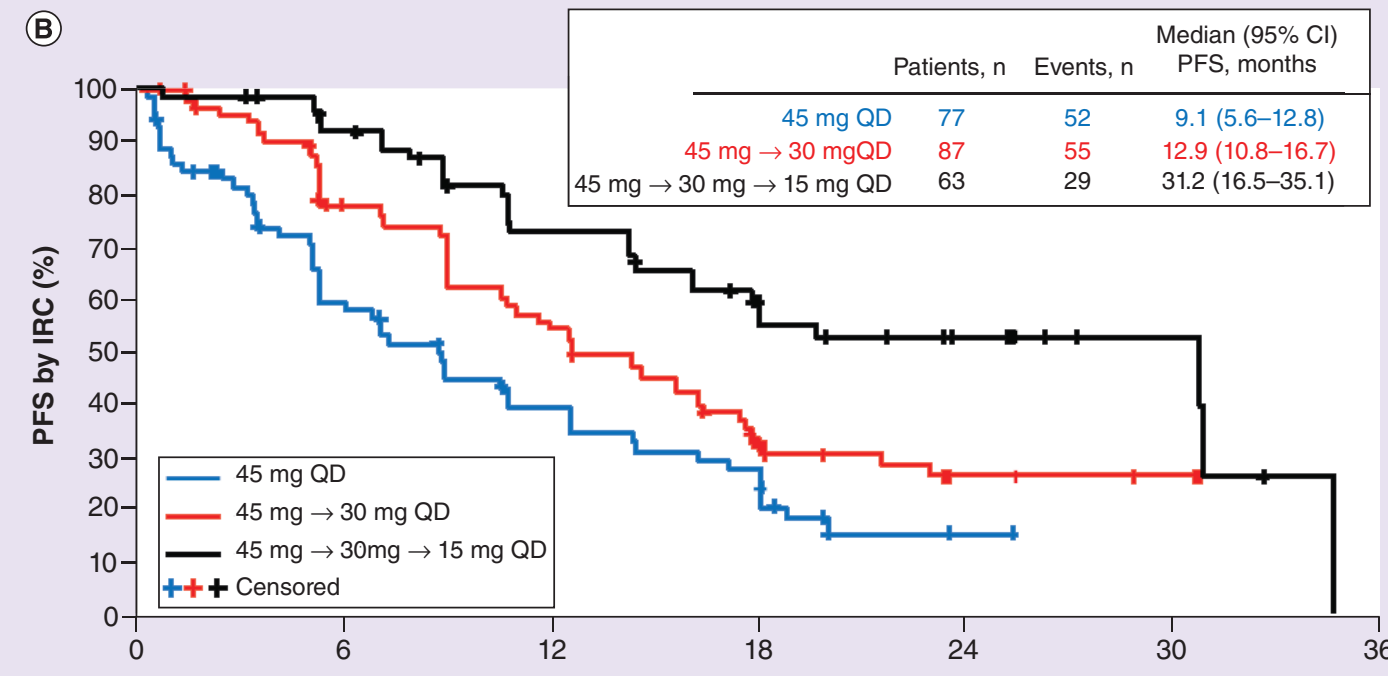

No. at risk:

$45 \mathrm{mg}$ QD

$45 \mathrm{mg} \rightarrow 30 \mathrm{mg}$ QD

$45 \mathrm{mg} \rightarrow 30 \mathrm{mg} \rightarrow 15 \mathrm{mg}$ QD

77
87
63

38
61
55

$\begin{array}{ll}38 & 23 \\ 51 & 43 \\ 55 & 40\end{array}$

Time (months)

Figure 1. Progression-free survival for dacomitinib in all patients, those with dose reduction and by lowest dose. (A) Kaplan-Meier curves for estimated PFS for all patients treated with dacomitinib (blue line; $n=227$ ) and patients who dose reduced to either 30 mg QD or $15 \mathrm{mg}$ QD as lowest dose (green line; $\mathrm{n}=150$ ). (B) Kaplan-Meier curves for estimated PFS for patients who remained on $45 \mathrm{mg}$ QD dacomitinib (blue line; $n=77$ ), patients who received $30 \mathrm{mg}$ QD as lowest dose (red line; $\mathrm{n}=87$ ) and patients who received $15 \mathrm{mg}$ QD as lowest dose (black line; $n=63$ ). PFS was defined as the time from randomization to objective disease progression or death from any cause, whichever occurred first. Vertical lines on the survival curves indicate censoring of data.

IRC: Independent review committee; NR: Not reached; PFS: Progression-free survival; QD: Once daily. 


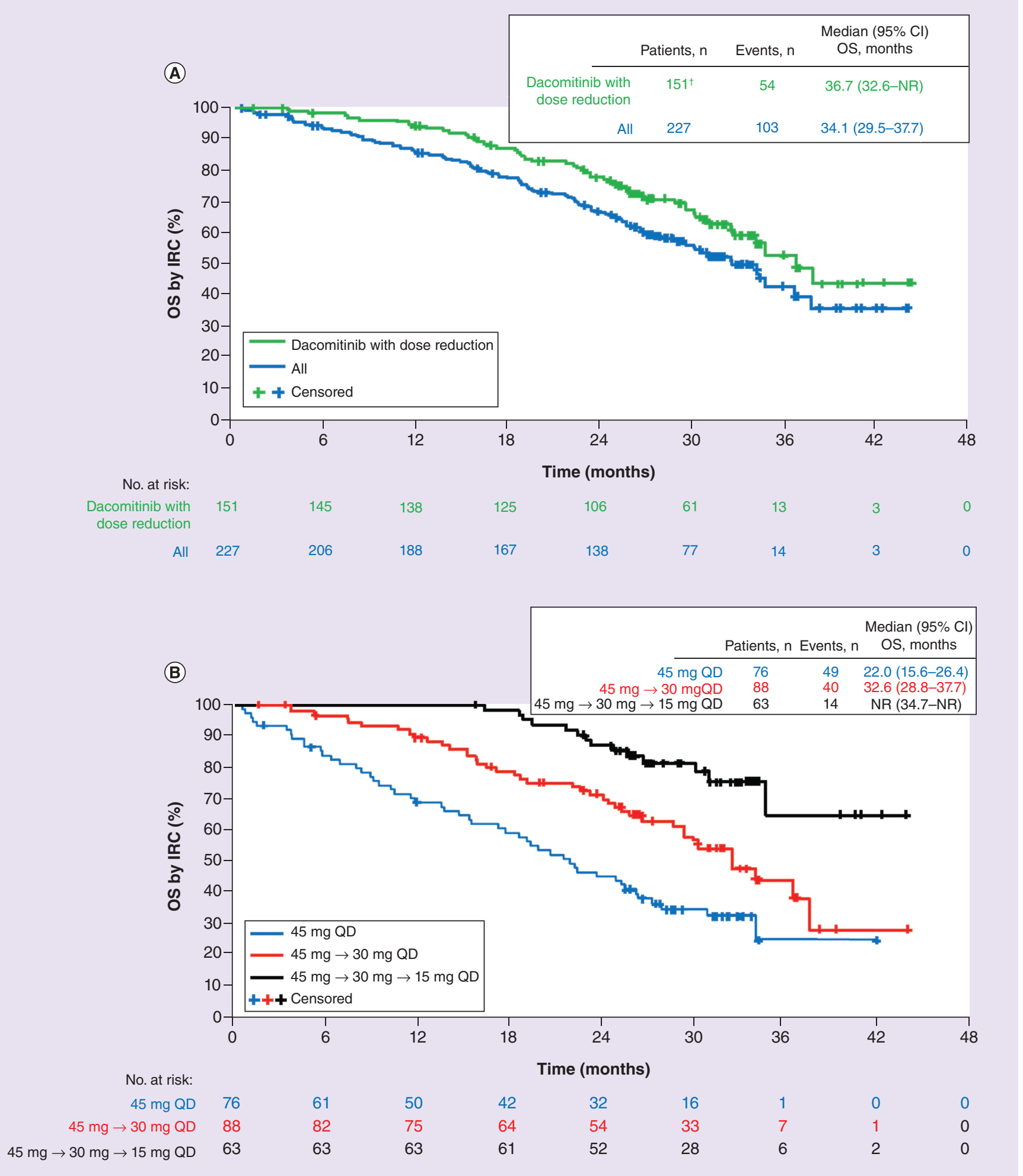

Figure 2. Overall survival for dacomitinib in all patients, those with dose reduction and by lowest dose. (A) Kaplan-Meier curves for estimated OS for all patients treated with dacomitinib (blue line; $n=227$ ) and patients who dose reduced to either $30 \mathrm{mg} Q D$ or $15 \mathrm{mg}$ QD as lowest dose (green line; $n=151$ ). (B) Kaplan-Meier curves for estimated OS for patients who remained on 45 mg QD dacomitinib (blue line; $\mathrm{n}=76$ ), patients who received $30 \mathrm{mg}$ QD as lowest dose (red line; $\mathrm{n}=88$ ), and patients who received $15 \mathrm{mg}$ QD as lowest dose (black line; $n=63$ ). OS was defined as the time from randomization to death from any cause. Vertical lines on the survival curves indicate censoring of data.

${ }^{\dagger} n=151$ as opposed to 150 due to later cut-off date for OS (17 February 2017) than for other end points (29 July 2016). IRC: Independent review committee; NR: Not reached; OS: Overall survival; QD: Once daily. 


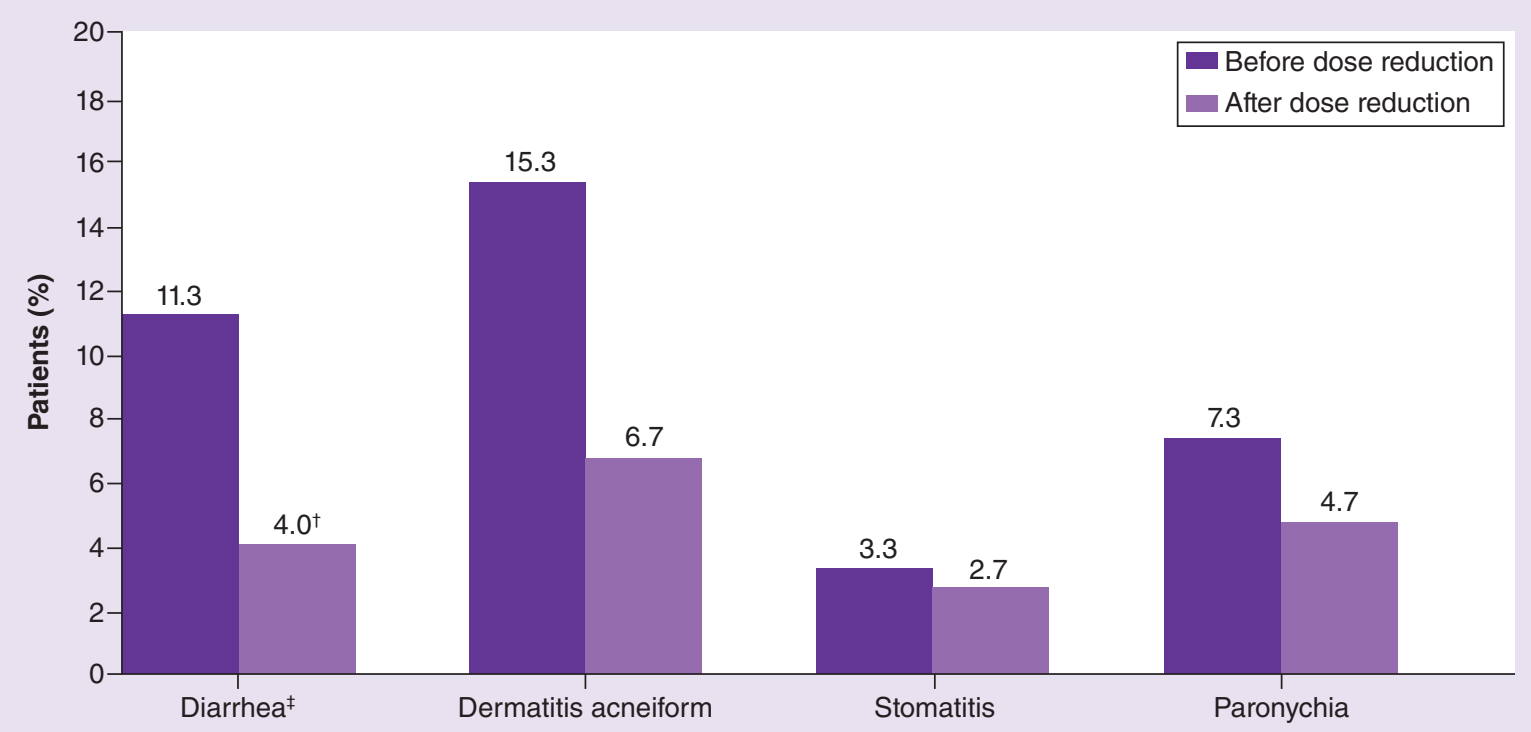

Figure 3. Grade 3 adverse events with dose reduction. Shown are incidence of grade 3 AEs of interest before (dark purple bars) and after (lighter purple bars) dose reduction in the population of all patients experiencing a dose reduction due to an $A E(n=150)$. There were no grade 4 events of interest in dacomitinib-treated patients who had a dose reduction.

†One grade 5 event occurred after dose reduction and is not included in this percentage.

$¥$ Clustered term encompassing acute prerenal failure, azotemia, dehydration, diarrhea, blood urea nitrogen/creatinine ratio increased, electrolyte imbalance, hypovolemia and prerenal failure.

$\mathrm{AE}$ : Adverse event.

\section{Safety}

Among patients who dose reduced, all patients $(\mathrm{n}=150 ; 100 \%)$ experienced all-causality AEs prior to DR; 37 (24.7\%) patients who dose reduced did so due to a grade $3 \mathrm{AE}$. The most common AEs leading to DR were skin toxicities $(\mathrm{n}=94 ; 62.7 \%)$, dermatitis acneiform $(\mathrm{n}=46 ; 30.7 \%)$, paronychia $(\mathrm{n}=38 ; 25.3 \%)$ and diarrhea $(\mathrm{n}=21$; $14.0 \%)$. The most common all-grade AEs in patients who subsequently underwent DR ( $\mathrm{n}=150)$ were diarrhea $(\mathrm{n}=132 ; 88.0 \%)$, paronychia $(\mathrm{n}=82 ; 54.7 \%)$, dermatitis acneiform $(\mathrm{n}=76 ; 50.7 \%)$ and stomatitis $(\mathrm{n}=69$; $46.0 \%$ ). The incidence of grade 3 AEs of interest (diarrhea, dermatitis acneiform, stomatitis and paronychia) decreased after DR (Figure 3). Notably, incidence of grade 3 diarrhea decreased from $11.3 \%(\mathrm{n}=17)$ to $4.0 \%$ $(\mathrm{n}=6)$ and that of dermatitis acneiform decreased from $15.3 \%(\mathrm{n}=23)$ to $6.7 \%(\mathrm{n}=10)$. There were no grade 4 events of interest in patients who had a DR. The incidence and severity of individual AEs of interest generally decreased after DR (Figure 4). However, incidence and severity did not decrease in all cases. For example, while the incidences of grade 3 dermatitis acneiform and paronychia decreased after DR, the incidence of grade 2 events did not. Incidence of grade 2 and grade 3 diarrhea (Figure 4A), and grade 2 and grade 3 stomatitis (Figure 4C) decreased after DR irrespective of whether $30 \mathrm{mg}$ QD or $15 \mathrm{mg}$ QD was the lowest dose. Permanent discontinuations due to treatment-related AEs occurred in $22(9.7 \%)$ patients treated with dacomitinib.

\section{Pharmacokinetic analyses}

A total of 177 patients had available $C_{\text {trough,ss }}$ data at cycle 2, day 1. Patients who received the $45 \mathrm{mg}$ QD dacomitinib dose without any DR $(n=63)$ tended to have lower initial plasma dacomitinib exposure, as measured by dacomitinib $\mathrm{C}_{\text {trough,ss }}$ at cycle 2, day 1 than those who dose reduced to either $30 \mathrm{mg}$ QD ( $\mathrm{n}=67$ ) or $15 \mathrm{mg}$ QD ( $\mathrm{n}=47)$ (Figure 5). Geometric mean (geometric coefficient of variation; gCV\%) of $\mathrm{C}_{\text {trough,ss }}$ at cycle 2, day 1 was $59.1 \mathrm{ng} / \mathrm{ml} \mathrm{(35 \% )} \mathrm{for} \mathrm{patients} \mathrm{without} \mathrm{any} \mathrm{DR,} 67.7 \mathrm{ng} / \mathrm{ml}(38 \%)$ for patients with $30 \mathrm{mg}$ QD as the lowest dose, and $67.6 \mathrm{ng} / \mathrm{ml}(61 \%)$ for patients with $15 \mathrm{mg}$ as the lowest dose, respectively.

\section{Discussion}

The ARCHER 1050 trial showed significant improvement in PFS and OS for dacomitinib versus gefitinib, and in the $66.1 \%(\mathrm{n}=150)$ patients who had dose adjustments the efficacy was maintained $[2,3]$. Toxicity management 


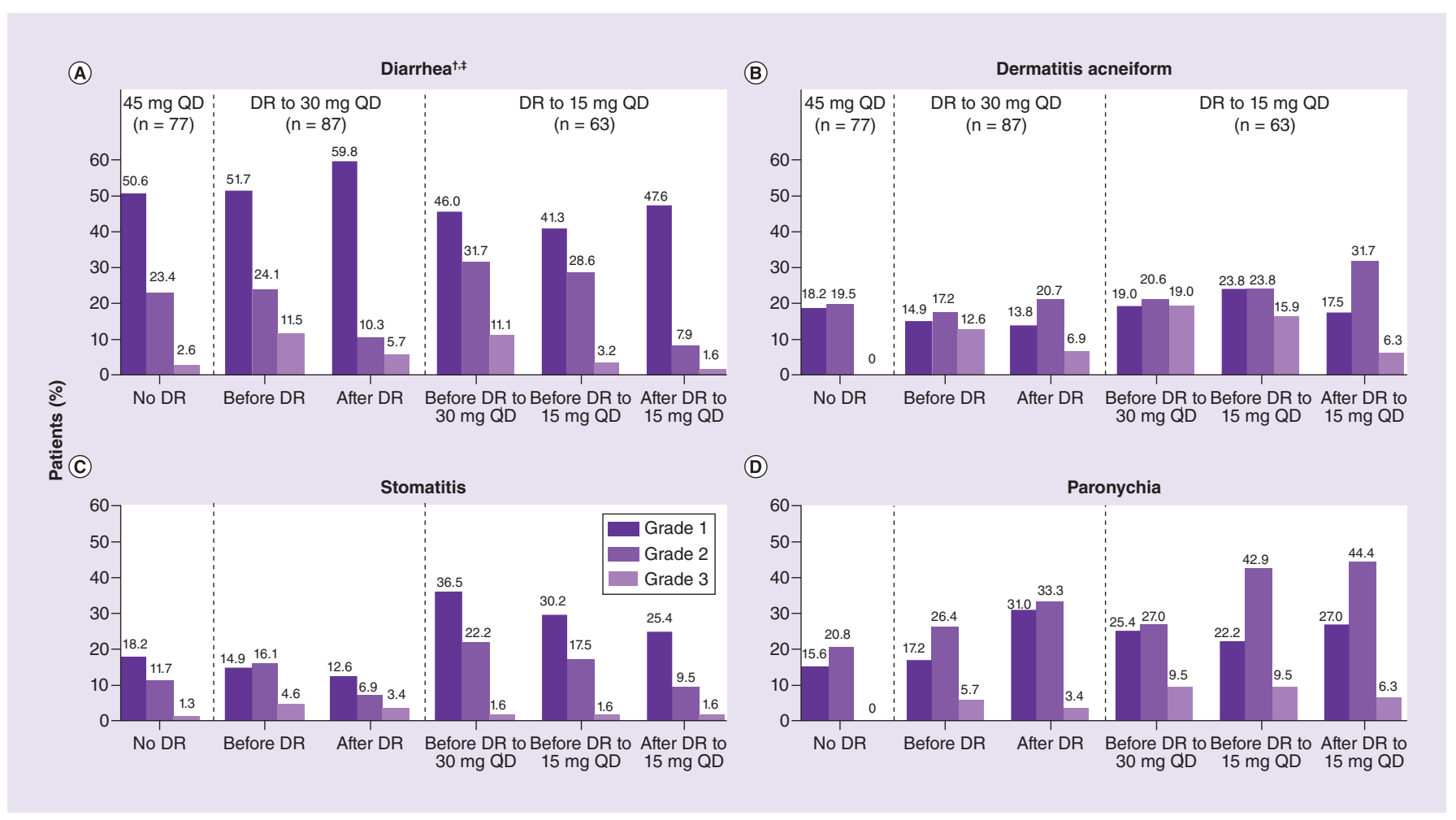

Figure 4. Safety by the lowest dacomitinib dose. Shown on the left side of each panel (A-D) are patients in the as-treated population $(n=227)$ who stayed on the $45 \mathrm{mg}$ QD dacomitinib dose (dark purple bars; $n=77)$, in the middle are patients (before and after dose reduction) who received a lowest dose of $30 \mathrm{mg}$ QD (lighter purple bars; $\mathrm{n}=87$ ), and on the right are patients (before and after dose reduction first to $30 \mathrm{mg} \mathrm{QD}$, then to $15 \mathrm{mg} Q D$ ) who received a lowest dose of $15 \mathrm{mg}$ QD (lightest purple bars; $n=63$ ).

$\dagger$ Includes one grade 4 event in a patient who did not have a dose reduction.

$¥$ Clustered term encompassing acute prerenal failure, azotemia, dehydration, diarrhea, blood urea nitrogen/creatinine ratio increased, electrolyte imbalance, hypovolemia and prerenal failure.

DR: Dose reduction; QD: Once daily.

is a challenge across the entire class of EGFR TKIs, with the majority of drug-related AEs mediated by the inhibition of epithelial EGFR expression [6,7]. The most commonly reported AEs with dacomitinib treatment (including diarrhea, dermatitis acneiform, stomatitis and paronychia) both in the ARCHER 1050 trial [2], as well as previous dacomitinib trials [8-10], are associated with this mechanism. With regard to second-generation TKIs, skin and gastrointestinal toxicities related to this mechanism are the major reasons for dose modification and discontinuation [2,11]. Permanent discontinuation due to treatment-related AEs occurred in $9.7 \%(\mathrm{n}=22)$ of patients treated with dacomitinib in the ARCHER 1050 trial and in 6.3\% $(\mathrm{n}=15)$ of patients treated with afatinib in the LUX-Lung 6 trial [4]. The DR rates for these treatment groups, meanwhile, were $66.1 \%(\mathrm{n}=150)$ and 28.0\% ( $\mathrm{n}=67)$ in ARCHER 1050 and LUX-Lung 6, respectively [4]. However, this safety profile comparison should be made with caution for several reasons. First, LUX-Lung 6, in contrast to ARCHER 1050, was conducted exclusively in an Asian patient population. Additionally, cross-trial comparison is difficult in general due to potential differences in a number of other variables (e.g., trial design details, age of patients, disease stage and disease subtype).

The current analysis revealed that dose modifications helped to manage AEs without the need for permanent discontinuation in the majority of patients, while maintaining efficacy. Additionally, dacomitinib DRs decreased the incidence and severity of all-causality AEs. Notably, the incidence of grade 2 and grade 3 diarrhea and stomatitis, two AEs with higher grade incidence at the starting dose of $45 \mathrm{mg}$ QD in ARCHER 1050, decreased after DR. While the incidence of grade 3 dermatitis acneiform and paronychia also decreased after DR, the incidence of grade 2 events did not. We hypothesize that the increase seen in grade 2 dermatitis acneiform events following DR may in part be a result of grade 3 events converting to grade 2 events. The exact reason for the observed increase in grade 2 paronychia events following $\mathrm{DR}$ is unclear. 


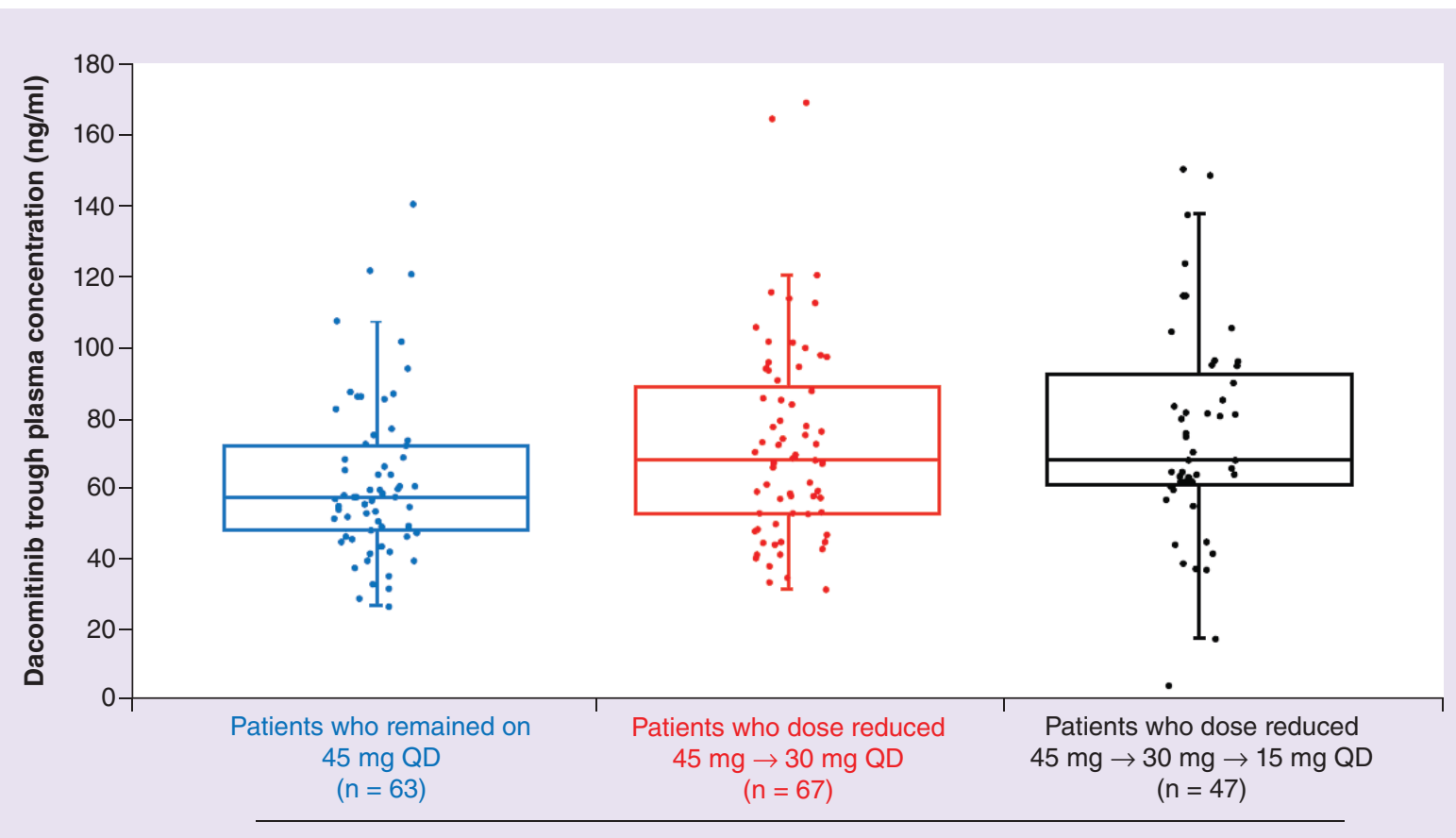

Measurement at cycle 2, day 1

(all patients received $45 \mathrm{mg}$ QD dacomitinib dose at measurement)

Figure 5. Comparison of dacomitinib initial exposure between lowest dose groups after first treatment cycle. Shown is a comparison of trough plasma concentrations on day 1 of cycle 2 in patients remaining on dacomitinib 45 mg QD (blue symbols; $n=64$ ), dose reducing to $30 \mathrm{mg}$ QD (red symbols; $\mathrm{n}=74$ ) as lowest dose, or dose reducing to $15 \mathrm{mg}$ QD as lowest dose (black symbols; $n=47$ ). All patients received $45 \mathrm{mg}$ QD dacomitinib at the time of measurement. Boxes represent the median and interquartile range; the whiskers represent the 10th and 90 th percentiles and the dots show data points outside percentiles.

QD: Once daily.

The PK analysis showed that patients who remained at $45 \mathrm{mg}$ QD tended to have the lowest initial dacomitinib exposure, compared with those patients who had their dose reduced to $30 \mathrm{mg}$ QD or $15 \mathrm{mg}$ QD as the lowest dose. These results are similar to those seen with the second-generation TKI afatinib, where, at day 22 , the patient group that would remain on the starting dose (40 mg QD) until day 43 had lower exposure than the group that had the dose reduced (to $30 \mathrm{mg}$ QD) by day 43 [4]. Also, similar to results in the present report, patients whose dose reduced to $30 \mathrm{mg}$ QD afatinib in the first 6 months of treatment had median PFS comparable with that in patients remaining on the $40 \mathrm{mg}$ QD starting dose [4]. Coupled with the fact that there are no apparent baseline characteristics predictive of which patients will eventually have a DR, the exposure data in the current report are not sufficient to determine whether starting on a lower dose (e.g., $30 \mathrm{mg}$ QD) could be appropriate. Further studies, potentially in conjunction with modeling and simulations based on available clinical data, are needed to assess if, and for which patients, a lower starting dose might be prudent. In this regard, it should be noted that starting an EGFR TKI at a lower dose is common in real-world clinical practice. One recent study with afatinib showed that starting patients at a lower dose (31\% started on less than the standard starting dose of $40 \mathrm{mg}$ QD) did not result in compromised efficacy [5].

When interpreting the PFS and OS results summarized by the lowest dose received, it is important to recognize that comparisons across lowest dose levels of patients who dose reduced are confounded by the fact that the lowest dose received was a study outcome, and therefore, PFS and OS results by lowest dose received were outcome driven and not based on criteria present at randomization, as all patients received $45 \mathrm{mg}$ QD as the starting dose. Further, the time to each successive DR was not immediate. The median time to DR for the 87 patients who dose reduced to $30 \mathrm{mg}$ QD as the lowest dose received was 12.9 weeks, while for the 63 patients who subsequently dose reduced to $15 \mathrm{mg}$ as the lowest dose received, median time to the first DR (to $30 \mathrm{mg}$ QD) was 8.4 weeks and median time to the subsequent DR to $15 \mathrm{mg}$ QD (from $30 \mathrm{mg}$ QD) was 12.4 weeks. It is important to note that nonresponding patients with short treatment duration were less likely to have undergone DR at all. Since all patients started on 
the $45 \mathrm{mg}$ dose, and patients who eventually had their dose reduced remained on $45 \mathrm{mg}$ QD for some time before $\mathrm{DR}$, no conclusions can be drawn as to the potential efficacy of starting doses of either $30 \mathrm{mg}$ QD or $15 \mathrm{mg}$ QD, and direct statistical comparisons between the presented DR groups would not be informative with respect to those starting doses. A limitation of the study is that no conclusions can be drawn concerning the potential effect of DRs on the progression or control of brain metastases, since patients with brain metastases were excluded from participation in ARCHER 1050.

\section{Conclusion}

In summary, we found that the incidence and severity of AEs declined following DR (e.g., grade 3 dermatitis acneiform was reduced from 15.3 to 6.7\%). However, both median PFS (14.7 and 16.6 months) and median OS (34.1 and 36.7 months) were similar in all dacomitinib-treated patients and those with DR. Thus, tolerabilityguided dose modifications enabled patients to continue with dacomitinib treatment and benefit from improvement in PFS and OS.

\section{Summary points}

- Dose modifications decreased the incidence and severity of treatment-related adverse events.

- Initial plasma dacomitinib exposure at $45 \mathrm{mg}$ QD was generally lower in patients remaining at $45 \mathrm{mg}$ QD compared with dose-reducing patients.

- The benefit of progression-free survival (PFS) and overall survival (OS) was maintained in patients who received dose reductions.

- Both median PFS and median OS were similar in all dacomitinib-treated patients and those with dose reduction.

- Tolerability-guided dose modifications enabled patients to manage AEs and continue with dacomitinib treatment and benefit from improvement in PFS and OS.

\section{Supplementary data}

To view the supplementary data that accompany this paper please visit the journal website at: www.futuremedicine.com/doi/sup $\mathrm{pl} / 10.2217 /$ fon-2019-0299

\section{Authors' contributions}

All authors contributed to the concept and design of the study, to conducting the analysis, interpretation of the results, writing and revisions of the manuscript and approval of the final version submitted for publication.

\section{Acknowledgments}

We would like to thank all ARCHER 1050 patients and their families, participating clinical sites, all the investigators, nurses and site staff, Pfizer Inc., and SFJ Pharmaceuticals ${ }^{\circledR}$.

Financial \& competing interests disclosure

TS Mok reports receiving fees for speaking/advisory board roles, receiving grant/research support, and receiving honoraria from AstraZeneca, Boehringer Ingelheim, Bristol-Myers Squibb, Novartis, and Pfizer, reports both receiving fees for speaking/advisory board roles and grant/research support from Clovis Oncology and SFJ Pharmaceuticals ${ }^{\circledR}$, reports both receiving fees for speaking/advisory board roles and receiving honoraria from Eli Lilly, Genentech, and Merck Sharp \& Dohme, reports both receiving fees for speaking/advisory board roles and owning stock in Cirina, reports receiving fees for speaking/advisory board roles from ACEA Biosciences, Celgene, Cirina, GeneDecode, Ignyta, Merck Serono, Oncogenex, and Vertex, reports receiving grant/research support from Eisai, Roche, and Taiho Pharmaceutical, and reports owning stock in Sanomics. K Nakagawa reports both receiving grant/research support and honoraria from Astellas Pharma, AstraZeneca, Boehringer Ingelheim, Bristol-Myers Squibb, Chugai Pharmaceuticals, Daiichi Sankyo, Eli Lilly, EPS Holdings, Kyowa Hakko Kirin Co, Novartis, Ono Pharmaceuticals, Pfizer, and Taiho Pharmaceuticals, reports receiving grant/research support from AbbVie, AC Medical, Eisai, GlaxoSmithKline, Japan Clinical Research Operations, Merck Serono, Otsuka Pharmaceuticals, Parexel International, PPD-SNBL, Takeda Pharmaceuticals, and Yakult Honshu Co., and reports receiving honoraria from Ayumi Pharmaceuticals, Kissei Pharmaceuticals, Quintiles, Showa Yakuhin Kako Co. and SymBio Pharmaceuticals. MR Migliorino reports receiving fees for speaking/advisory board roles from AstraZeneca, Boehringer Ingelheim and Bristol-Myers Squibb. A Pluzanski reports both receiving speaker bureau fees and receiving fees for 
speaking/advisory board roles from AstraZeneca, Boehringer Ingelheim, Bristol-Myers Squibb, Merck Sharpe \& Dohme, and Roche. Y-L Wu reports receiving fees for speaking/advisory board roles, receiving grant/research support, and receiving honoraria from Boehringer Ingelheim and Roche, reports both receiving fees for speaking/advisory board roles and receiving honoraria from AstraZeneca, and reports receiving honoraria from Eli Lilly and Sanofi. R Linke is an employee of SFJ Pharmaceuticals ${ }^{\circledR}$. G Devgan, $S$ Quinn, W Tan and T Wang are employees of and own stock in Pfizer. This study was funded by Pfizer and SFJ Pharmaceuticals ${ }^{\circledR}$. The authors have no other relevant affiliations or financial involvement with any organization or entity with a financial interest in or financial conflict with the subject matter or materials discussed in the manuscript apart from those disclosed.

Editorial support was provided by JN Raksin, PhD, inScience Communications, Springer Healthcare (Philadelphia, PA, USA) and funded by Pfizer Inc.

Ethical conduct of research

The authors state that they have obtained appropriate institutional review board approval or have followed the principles outlined in the Declaration of Helsinki for all human or animal experimental investigations. In addition, for investigations involving human subjects, informed consent has been obtained from the participants involved.

\section{Data sharing statement}

The authors certify that this manuscript reports the secondary analysis of clinical trial data that have been shared with them, and that the use of this shared data is in accordance with the terms (if any) agreed upon their receipt. The source of this data is: NCT01774721. Upon request, and subject to certain criteria, conditions, and exceptions (see www.pfizer.com/science/clinical-tr ials/trial-data-and-results for more information), Pfizer will provide access to individual deidentified participant data from Pfizersponsored global interventional clinical studies conducted for medicines, vaccines and medical devices for indications that have been approved in the USA and/or European Union or in programs that have been terminated (i.e., development for all indications has been discontinued). Pfizer will also consider requests for the protocol, data dictionary and statistical analysis plan. Data may be requested from Pfizer trials 24 months after study completion. The deidentified participant data will be made available to researchers whose proposals meet the research criteria and other conditions, and for which an exception does not apply, via a secure portal. To gain access, data requestors must enter into a data access agreement with Pfizer.

\section{Open access}

This work is licensed under the Attribution-NonCommercial-NoDerivatives 4.0 Unported License. To view a copy of this license, visit http://creativecommons.org/licenses/by-nc-nd/4.0/

\section{References}

Papers of special note have been highlighted as: $\bullet$ of interest or $\bullet \bullet$ of considerable interest

1. Engelman JA, Zejnullahu K, Gale CM et al. PF00299804, an irreversible pan-ERBB inhibitor, is effective in lung cancer models with EGFR and ERBB2 mutations that are resistant to gefitinib. Cancer Res. 67(24), 11924-11932 (2007).

2. Wu YL, Cheng Y, Zhou X et al. Dacomitinib versus gefitinib as first-line treatment for patients with EGFR-mutation-positive non-small-cell lung cancer (ARCHER 1050): a randomized, open-label, Phase III trial. Lancet Oncol. 18(11), 1454-1466 (2017)

-. Improvement in progression-free survival with dacomitinib over gefitinib in patients with EGFR mutation-positive non-small-cell lung cancer (NSCLC) was demonstrated in this randomized Phase III ARCHER 1050 study.

3. Mok TS, Cheng Y, Zhou X et al. Improvement in overall survival in a randomized study that compared dacomitinib with gefitinib in patients with advanced non-small-cell lung cancer and EGFR-activating mutations. J. Clin. Oncol. 36(22), 2244-2250 (2018).

-. Significant improvement in overall survival compared with the standard-of-care TKI, gefitinib, in this mature overall survival analysis from the Phase III ARCHER 1050 study.

4. Yang JC, Sequist LV, Zhou C et al. Effect of dose adjustment on the safety and efficacy of afatinib for EGFR mutation-positive lung adenocarcinoma: post hoc analyses of the randomized LUX-Lung 3 and 6 trials. Ann. Oncol. 27(11), 2103-2110 (2016).

-• Post hoc analysis of data from treatment-naive patients with advanced EGFR mutation-positive NSCLC, in two Phase III studies (LUX-Lung 3/6), showed that tolerability-guided dose adjustment effectively reduced afatinib-related adverse events without affecting therapeutic efficacy.

5. Halmos B, Tan EH, Soo RA et al. Impact of afatinib dose modification on safety and effectiveness in patients with EGFR mutation-positive advanced NSCLC: results from a global real-world study (RealGiDo). Lung Cancer 127, 103-111 (2019).

6. Hirsh V, Blais N, Burkes R, Verma S, Croitoru K. Management of diarrhea induced by epidermal growth factor receptor tyrosine kinase inhibitors. Curr. Oncol. 21(6), 329-336 (2014).

7. Melosky B, Leighl NB, Rothenstein J, Sangha R, Stewart D, Papp K. Management of EGFR TKI-induced dermatologic adverse events. Curr. Oncol. 22(2), 123-132 (2015). 
- Basic strategies for management of EGFR TKI-associated acneiform rash, stomatitis and paronychia were detailed in this expert opinion review.

8. Ramalingam SS, Janne PA, Mok T et al. Dacomitinib versus erlotinib in patients with advanced-stage, previously treated non-small-cell lung cancer (ARCHER 1009): a randomized, double-blind, Phase III trial. Lancet Oncol. 15(12), 1369-1378 (2014).

9. Reckamp KL, Giaccone G, Camidge DR et al. A Phase II trial of dacomitinib (PF-00299804), an oral, irreversible pan-HER (human epidermal growth factor receptor) inhibitor, in patients with advanced non-small-cell lung cancer after failure of prior chemotherapy and erlotinib. Cancer 120(8), 1145-1154 (2014).

10. Ellis PM, Shepherd FA, Millward M et al. Dacomitinib compared with placebo in pretreated patients with advanced or metastatic non-small-cell lung cancer (NCIC CTG BR.26): a double-blind, randomised, Phase III trial. Lancet Oncol. 15(12), 1379-1388 (2014).

11. Wu YL, Zhou C, Hu CP et al. Afatinib versus cisplatin plus gemcitabine for first-line treatment of Asian patients with advanced non-small-cell lung cancer harbouring EGFR mutations (LUX-Lung 6): an open-label, randomized Phase III trial. Lancet Oncol. 15(2), 213-222 (2014). 
